\title{
Exploration and Reflection on the Research Method of Oral History of Tibetan Dance
}

\author{
Qiongqiong Lai ${ }^{1, *}$ \\ ${ }^{1}$ Shaanxi Xueqian Normal University, Xi'an, Shaanxi, China \\ "Corresponding author. Email: wdwhysyjzx@163.com

\begin{abstract}
Currently, the research on Chinese ethnic and folk dance has achieved fruitful results, but some fields still need to be further explored. The research method of oral history is feasible and irreplaceable in practice, which makes up for textual research. The study on the oral history of Tibetan dance is feasible and urgent, which is of great value to the understanding of minority dance culture and the protection of intangible cultural heritage. Specific research and practices are faced with such obstacles as language barrier, inaccurate translation, poor support from literature, and human interference, resulting in low efficiency or stagnation of interviews. Therefore, several issues in the study on the oral history of Tibetan dance should be carefully controlled, and scientific and efficient ways and methods should be proposed to solve the problems of "whom to talk to", "what to talk about" and "how to talk", so as to guarantee smooth study and unveil the mystery of Tibetan dance.
\end{abstract}

Keywords: Tibetan dance, research methodology of oral history, domain and attribute characteristics, research dilemma, key nodes

\section{INTRODUCTION}

Chinese ethnic and folk dance generally refers to "dance that is emerged and circulated among the folk, restricted by folk culture, improvised performance but relatively stable in style, with self-entertainment as the main function"1. The research on its history and dance aesthetics mainly relies on "investigation of local customs". The method has been adopted and inherited since the emergence of Chinese ethnic and folk dance, and has become one of the approaches to study the theories and methods of oral history with the development of theoretical research. The research method of oral history in ethnomusicology has been applied for a long time. Whether the upsurge of the compilation of music history in the first half of the $20_{\text {th }}$ century or the visiting, recording and sorting of folk songs in provinces and cities during the compilation of Chinese Folk Songs at the beginning of the People's Republic of China are important practice in the study of oral history. In recent years, scholars such as Feng Jicai, Liang Maochun and Xie Jiaxin have put forward diversified views on oral history of folk music. On November 30, 2017, Mr. Liang Maochun gave a lecture on Eight Methods of Musical Oral History in China Conservatory of Music, and talked with scholars as Xie

Gao Du, Huang Yihua. Dictionary of Teaching Terms in Chinese Ethnic and Folk Dance [M]. Shanghai: Shanghai Music Publishing House, 2014:6.
Jiaxing, Ding Xudong, explaining method elements as "pragmatic approach, historical perspective, topic selection and outline writing". Feng Jicai employed oral history in his Ten Years of One Hundred People, which laid the foundation of his oral history methodology. As for the oral history of Chinese ethnic and folk dance, in 2004, Professor Zhao Tiechun of Beijing Dance Academy began to pool practical experience of frontline educators and theoretical scholars through oral interviews. Later, Origin and Analysis of Traditional and Typical Combination of Chinese Ethnic and Folk Dance, Memory of Dance Journey - Oral History of a Chinese Ethnic and Folk Dance Educator and Oral History of Chinese Ethnic and Folk Dance were published successively. The research method of oral history has been adopted in these research results, and the personal experience of scholars has also become an important factor. The publication of these books also indicates that the research method of oral history has become an integral in the academic research field of Chinese ethnic and folk dance. Among them, research on the oral history of Tibetan traditional dance is still in its infancy. Constrained by various subjective and objective hampers, no systematic research results have been obtained. Therefore, the combination of the research methods of oral history and the exploration of Tibetan dance deserves attention. 


\section{BASIC CONCEPT CATEGORY AND CORE CONTENT OF ORAL HISTORY OF DANCE}

The basic concepts of oral history held by contemporary Chinese historians falls into two camps: "first, oral history is regarded as a new form of historiography, which is a branch of historiography parallel to the literal historiography with historical documents as research object. The second is to take oral history as a new historical method, that is, to collect and use oral historical materials in the way of recorded interview to study history" ${ }^{2}$. Despite their differences in concept, they share cognition of oral history. Therefore, the concept of study on oral history of dance can be considered as follows: the unique way of obtaining research data is applied, and the "primary data" can be obtained by mining the respondents' deep perception of dance culture and history, making up for the important content neglected in the current literature and archaeological research, restoring the development of folk dance, and thus forming a research perspective different from the previous ones. In terms of the research method and concept of dance oral history, the regular method of oral history can be used for reference. That is, "a form of historical study in which well-prepared interviewers collect and collate oral memories and historical perspectives by means of transcripts, tapes, etc."3. On this basis, considering the particularity of dance oral interview, the topic, content, method, communication context and other issues were adjusted to achieve favorable research results. Such communication by word of mouth is ubiquitous in the inheritance of human culture and history. It was not until the 1940s that a professional discipline developed in the United States. China introduced this research method in the 1980s and employed it in several fields of culturology.

The study on oral history of dance has formed a certain development momentum in China. Based on the existing research results of Chinese ethnic and folk dance and supplemented by the research method of oral history, this paper observes and thinks about the history and culture of dance from a new dimension. However, the integration of "oral history" and "dance" cannot accomplished in one move, and there are many problems to be solved in the specific research process. For example, how the orientation of the attribute and boundary of new discipline not only reflects independent research perspective but also integrates with the literature history research? What is the particularity of the interview when the research method of oral history is connected with the silent dance

Qi Linna. The Obscure Historical Corner - The Social Significance and Theoretical Contribution of "Oral History" [J]. Contemporary Cinema, 2015(11): 165

Ding Yizhuang, Wang Run. Readings in History: Oral History [M]. Beijing University Press, 2011:1. language? How to use the research method scientifically to highlight the special characteristics of the study of oral history of dance? How does the application of oral history expand existing results, provide fresh historical materials, and promote the innovation of historical research at the overall level? Some of these problems and assumptions need to be clarified first, and some need to be constantly reflected in practice.

In view of the current cognition of dance oral history, the academic circle has widely recognized the achievements of practical research and the ability of oral history theory system. First, there is a consensus on the existence value of oral history of dance. Oral history of dance is a highly practical research method. Oral interviews with dance creators, performers, theoretical researchers, folk artists and bystanders were conducted to collect research materials, and the reliability of the content was proved by comparing existing literature with materialized archaeological results. Second, there is a consensus on the independent characteristics of the research methods of dance oral history. The study of oral history of dance can serve as historical documents to form a comprehensive, objective, detailed and multidimensional academic system in the context of contemporary research. With the development of research technology and depth on music history, the existing documents and historical materials often encounter problems of contradiction or insufficient evidence. Static data text, however, is "dumb". Therefore, the application of oral history is needed to provide more active material content and create evidence with "sense of presence". In the process, the audio and video can be used to extract historical materials from the speech and behavior of the inheritor, which is precious, and cannot be endowed by text historical materials. Third, there is a consensus on the logic of dance oral history. The key to the oral history of dance lies in the interview. The interview should be original, scientific, typical and diversified. Various opinions and wisdom should be taken into account to present an objective picture of history in all directions. As the object of oral history, researchers must have outstanding ability of research summary, sorting and reasoning, so as to flag the most valuable part of the interview and make up for the "blind spot" of current historical research. With rigorous academic attitude, the results of oral history are annotated with footnotes, marginal notes and endnotes, so that every research result can be reflected according to the facts. Fourth, there is a consensus on the complexity and difficulty of research on dance oral history. Compared with other cultural or artistic fields, the study of oral history of dance is very special, professional and abstract. Therefore, this is a challenging research work for researchers, who must master abundant research accumulation for support and fully prepare the 
interview content. Communication ability, language expression ability, logical thinking ability and resilience are essential for both parties to achieve the best communication state.

The development of Chinese ethnic and folk dance has a history of thousands of years and its long development and evolution relies on the cooperation of inheritance and communication. In addition to the dance works themselves, the study of minority dances also includes the foundation of dance culture, the creation and performance of dance concepts and the social feedback to the concepts, so as to form a complete cycle chain. Oral history of dance is an era in which the society pays attention to the results of works and provides dancers and inheritors with a platform to make their voices heard. The process of dance from scratch and the source of its charm in their eyes are narrated.

\section{DIFFICULTIES IN THE APPLICATION OF RESEARCH METHODS OF ORAL HISTORY OF TIBETAN DANCE}

The Tibetans are an ancient minority with splendid culture and history. Most people live on the QinghaiTibet Plateau, known as the "roof of the world". Nourished by special geography, history, climate, religion, folk customs and other comprehensive factors, the people here are simple, kind, hard-working and enthusiastic, with innate talent for singing and dancing. Dance, a "necessity" in religious ritual activities and a cultural carrier for the masses to amuse themselves, enjoys a strong religious flavor and numerous colors. There are many types of dance with cultural functions and connotations. Each dance serves as the epitome of Tibetan cultural life, and also reflects the unique dance aesthetic. Since the peaceful liberation of Tibet, many scholars have been keen on the observation and research of Tibetan ethnic and folk dance. However, there is still room for further exploration in historiography, aesthetics, posture, communication and culturology. With mature theoretical research methods and technical support, the research method of oral history interview on Tibetan dance can make up for the deficiency. However, in the current research process, the following difficulties are found:

First, the language barrier in oral history interview is difficult to overcome. If the field investigation of Tibetan dance is to get rich fruits, it must go deep into Tibetan cultural life. The Tibetan national language enjoys strong independence, and the dialects of different habitats have subtle differences. For most Han scholars, the language barrier is one of the most serious obstacles in the study of oral history. Some scholars can only overcome the difficulties through language translation. During the interview, it was found that many interviewees (inheritors) of Tibetan dance were old. Although there are translators proficient in Chinese and Tibetan, there are differences in word selection, mood and deep semantics between the semantic expressions of ethnic minority languages and posttranslation Chinese expressions. On the other hand, even though translation can narrow the gap in language, most translators themselves lack professional knowledge of dance, so it is difficult for them to fully express the core information about Tibetan dance in discourse, which leads to oral history investigation becoming common chat communication. Translating the results of such interviews into text would obviously leave out important information.

Secondly, the relative lack of literature restricts the oral history interview. Since the founding of the People's Republic of China, great progress has been made in the study on Tibetan culture, history, music and dance. However, there are still some deficiencies in the history of folk customs and of regional religions. The knowledge system of the interviewee may not be comprehensive. Therefore, there exists "fact-oriented" situation in the communication process. Every Tibetan dance is conceived and passed down from generation to generation in certain cultural opportunities. In oral communication, the collection of historical information often takes the macro cultural perspective as the entry point, which is closely connected with local religious beliefs, ritual activities and national festival culture. Without sufficient literature support, oral interview will lose the basis of dialogue between subject and object. Many useful language information is ignored due to the difference in level, and the interview results will be greatly reduced.

Third, the oral history told by the inheritor is not completely true, comprehensive and objective. During the interview of oral history of Tibetan dance, the interviewees should be carefully selected. They are creators, performers, educators or witnesses of a dance culture and have first-hand information resources and insights. However, in the interview practice, some uncontrollable "artificial" factors are also found. For example, the relatively narrow prejudices of the interviewed inheritors; partial understanding of some areas of Tibetan dance; and the idea of dance culture formed by the influence of the culture of the tribe or region is contrary to what the literature shows. In addition, the unspoken words in the communication process caused by national cultural concepts, religious beliefs and personal character. These problems exist in oral history interviews, which lead to the distortion of many data and variables too high to obtain accurate information.

Fourth, the academic quality of scholars is uneven, which also leads to the "overflow" of oral interview information. Strictly speaking, the study of oral history is a complex subject based on the interview results, 
forming a written data system, and then conducting indepth academic research and elaboration. It is not only the intuitive information obtained from the oral interview that can lead to a certain conclusion. It should not only pool ideas, but also conduct comparative research with literature materials, and publish research results or scientific opinions in open channels in a responsible academic spirit. However, there are some "unhealthy tendencies" in the field of oral history research. Some people unilaterally equate "oral history interview" with "news interview", and release some new information without further research. Especially in the current environment with advanced mobile Internet technology and far-reaching influence of "we media", some people lack the spirit of research and are too impetuous. They take the interview information out of context and quickly release it to the media for publicity. Many interviews "exaggerated", resulting in misleading public opinion. This obviously does more harm than good to the inheritance and development of Tibetan dance.

It can be seen that there are many practical difficulties to be overcome in the oral history study of Tibetan dance. On the one hand, the design of research methods should strictly follow the rules and logic of oral history; On the other hand, it is necessary to finetune the discussion on Tibetan dance and the actual situation in field investigation. It is possible to obtain the expected research benefits only by making adequate preparation and "tailoring".

\section{THE KEY NODE OF RESEARCH METHODS OF ORAL HISTORY OF TIBETAN DANCE}

The research process of oral history "is an academic activity that includes a series of behavioral steps, such as topic selection, rebound, dictation, recording, text arrangement, and writing publication" ${ }^{4}$. As for the topic selection of the Tibetan dance oral history study, it should focus on the researcher's "ability" and give consideration to the research innovation and value. After selecting the topic, an outline of the plan related to the interview should be prepared. In addition to the content, objectives, methods and steps at the research level, itinerary planning, contact with interviewees, appointment of interview time and provision of interview plan should also be considered. In addition to the basic methodology, the following key links should be paid attention to in the research on Tibetan dance:

First of all, the determination of interviewees is a prerequisite for the success of oral history of dance. In fact, as for the question of "who" is the target, it also reflects that the preparation of oral history research needs to be thorough and meticulous. There are various

\footnotetext{
Shan Jianxin. On the Concept, Nature and Method of Musical Oral History [J]. Music Research, 2015(04): 98.
}

types of Tibetan dance, with different genres and themes. The long historical evolution has gone through the initial period of singing and dancing, imitation of life and labor, functional period of religious rituals, and the period of dominating various blessing and praise activities after maturity. From the perspective of dance types, there are Nuo used in religious culture, Gaba influenced by Central Plains culture, "harmonic" of mass singing and dancing, and "zhuo" of both interest and skill. On the vast Tibetan Plateau, different cultural tribes differ in physical geography, living customs and their familiarity with various types of dances. Therefore, it is an important prerequisite to understand the history from whose mouth we should "pry" into the secrets of Tibetan dance. Traditionally, there are three types of interviewees to be sought: inheritors of dance art, witnesses of dance events, and experiencers of dance culture. In the eyes of the three types of interviewees, Tibetan dance has its own characteristics. The "reliability" and "validity" achieved by crossintegrating the information they say will obviously be excellent. Therefore, in the design process of interview plan, the researcher should take a certain type of Tibetan folk dance as the research target, mark the typical representative Tibetan dance source, consult the local county annals or relevant cultural and historical data, and find the interviewees with the most interview value, so as to get twice the result with half the effort. At the same time, according to the characteristics of oral history, attention should also be paid to the voice of ordinary people. In traditional historical studies, the upper class and elite figures are often the focus of writing, while the feelings and experiences of the lower class are easily ignored. The research method of oral history itself is a subversive historical research perspective, which collects the missing information in the real original ecological cultural environment. Therefore, emphasis should be placed on grassroots dance creators, performers and educators. For example, Tibetan dance educators of Minzu University of China, Ciren Sangmu and Danzhou Dorje, have been in the forefront of Tibetan dance education and communication for decades, and their observation and experience of Tibetan dance are bound to have the right of speech and representation.

Secondly, the interlocutor's communication ability and advance preparation is an important part to guarantee the interview quality. The interview method of oral history needs gentle and equal communication and many times of progressive and in-depth interview. But this is not the same as "small talk". The relationship between the researcher and the interviewee is very delicate. Only with rich knowledge, quick response and accurate entry point can researchers break the invisible barrier between host and guest, improve interview efficiency and enhance interview benefits. As for the horizontal interview content, the oral history interview 
should focus on mining rare, concrete and in-depth data, avoiding the overlap with the existing historical data research results, and reflecting a new dimension of information. This is very important for Tibetan dance research. Before the peaceful liberation of Tibet, Tibetan dance developed slowly under the special social system for a long time. After the liberation, most of the investigations of local customs focused on the macro level of cultural and artistic research. The lack of in-depth understanding of dance, coupled with objective reasons, led to some interviewees "can't say", "dare not say", "don't say much". The gradual opening of the society makes such "barrier" disappear gradually and brings a new opportunity for the study of oral history. Compared with the generality and abstraction of the traditional Tibetan dance more than 30 years ago, today it has formed the concrete type stratification. In the field of education, there are also "element teaching method", "combinational teaching method" and "Kumara" combination for Tibetan dance. In the absence of abundant documents, the research method of oral history is very precious. Therefore, the interview should seize the opportunity to talk more about the actual content, which is conducive to the inheritance and development of Tibetan dance.

In addition, sincere communication and in-depth understanding of the history of Tibetan dance are equally important. At the technical level, interviewing is all about people communicating. As for the technique design of oral history interview, many contents are listed in the existing books and research results. But the best way to open one's heart to anything is to be honest. The Tibetan people have lived on the plateau for generations, leading a diligent, honest and unsophisticated life. The current development of tourism and all kinds of media visits not only bring new life, but also "overdraw" the cultural environment of this place to some extent. Therefore, the oral history interview is better to give up the "routine" and adopt the "he who knows no tricks knows all tricks" method. Only by asking and listening attentively can people win the trust and respect of Tibetan compatriots and make them willing to give gifts without reservation. As for the "interview method", the particularity of the history of Tibetan dance should also be paid attention to. The Tibetan dance before liberation is mainly derived from the accumulation of folk culture, and religious dance is the most representative. After the liberation from 1957 to 1965 , Tibetan dance began the development and transformation of the "academy", drawing lessons from the "Soviet model" to form a folk dance system. In March 1979, after the end of the Cultural Revolution and at the beginning of the reform and opening up, Deng Xiaoping met with representatives of the Dalai Lama in Beijing. In 1980, the $10_{\text {th }}$ Panchen Lama was elected vice chairman of the National People's Congress. In May of the same year, Hu Yaobang, then
China's President, visited Tibet and brought with him many policies for the benefit of the people. Since 1984, Tibet has implemented the policy of "reform and opening up". The differences of political and economic environment in different historical stages have swayed the development of Tibetan dance. After the cultural Revolution, Tibetan dance was greatly promoted by the state cultural department. During this period, People's Daily published many articles like The Tibet Song and Dance Troupe has Concluded its Visit to Sweden ${ }^{5}$, Praise for the Tibetan Dance Drama Fairy Zhuowa Sangmu ${ }^{6}$, and The Tibet Autonomous Region Held its First Amateur Tibetan Opera Show ${ }^{7}$. Dance, an authoritative academic journal of dance in China, has also released Musical Notes ${ }^{8}$ and The Pearl of "Singing and Dancing" - Comment on Tibetan Dance Drama "Reba Love" . Therefore, the research on Tibetan oral history should clarify the content level of the interview so as to avoid time overlap and event confusion.

\section{CONCLUSION}

To sum up, the study of the oral history of Tibetan dance should adopt the methods in line with the study of the history of dance and the characteristics of Tibetan dance. Only by establishing the right interviewees, organizing the files in advance, and communicating with the interviewees with different identities, can researchers gain favorable research results. In the process of oral history research on Tibetan dance, various unexpected problems may be encountered. Some results from the "innate" limitation of oral history research, and some the special problems encountered by the unskilled operators. However, compared with the academic function and existence value of oral history itself, these difficulties and deficiencies are insignificant and can be overcome and solved through practice. It is believed that desirable research results can be obtained through unremitting efforts to uncover the deep cultural code of Tibetan dance.

\section{References}

[1] Liang Maochun, Liu Hehong. Ten Questions about Oral History of Chinese Music — The Lecture by Professor Liang Maochun

5 The Tibet Song and Dance Troupe has concluded its visit to Sweden [N]. People's Daily, 1978-10-4 (05).

6 Hu Guogang. Praise for the Tibetan dance drama Fairy Zhuowa Sangmu [N]. People's Daily, 1981-05-09 (08).

7 Xinhua News Agency. The Tibet Autonomous Region held its first amateur Tibetan Opera show [N]. People's Daily, 1980-06-02 (04).

8 Yang Shuming. Musical Notes [J]. Dance, 1981 (04): 37 38.

Hu Dade. The Pearl of "Singing and Dancing" — Comment on Tibetan Dance Drama "Reba Love" [J]. Dance, 1984 (03): 12-14. 
at China Conservatory of Music in May 2016 [J]. Journal of Tianjin Conservatory of Music. 2016(03):12-32. (in Chinese)

[2] Zhao Shufeng, Shan Jianxin. New Reflections on the Research on Oral History of Music [J]. Chinese Music. 2016(01): 195203. (in Chinese)

[3] Shan Jianxin. On the Concept, Nature and Method of Musical Oral History [J]. Music Research, 2015(04): 94-103. (in Chinese)

[4] Ding Xudong. The Theory and Practice Exploration of Modern Oral History and Musical Oral History - Summary and Reflection on The first National Symposium on Oral History of Music [J]. Journal of Xinghai Conservatory of Music, 2015(03): 145-152. (in Chinese)

[5] Liang Maochun. Talk on "The oral History of Music" [J]. Fujian Arts. 2014(04): 11-13. (in Chinese)

[6] Xiong Xiaohui. History and Logic of "Oral History": in the Development of Ethnic Music — Based on Study of Hereditary Chieftain Music of Tujia Nationality [J]. Journal of Northwest University for Nationalities. 2014(04): 87-96. (in Chinese) 